\title{
The LIDRAE water-Cherenkov air shower array
}

\section{Marcelo Augusto Leigui de Oliveira*,Vitor Prestes Luzio, Rafaela Wiklich Sobrinho, and Renan de Aguiar}

Center for Natural Sciences and Humanities (CCNH)

Federal University of $A B C$ (UFABC)

09210-580 Santo André - SP - Brazil

E-mail: leiguidufabc.edu.br

\begin{abstract}
We present the design, and preliminary results of the LIDRAE water-Cherenkov air shower array installed at UFABC $\left(23.6^{\circ} \mathrm{S}, 46.5^{\circ} \mathrm{W}, 750 \mathrm{~m}\right.$ a.s.l. $)$. LIDRAE detects the particles of extensive air showers with energies exceeding $100 \mathrm{TeV}$ and is able to measure the arrival direction and energy of the primary cosmic rays. The array is composed of three tanks each filled with one thousand liters of water with a large aperture photomultiplier on the top cover of each tank overlooking the water volume. The photomultipliers detect the Cherenkov light generated by the passage of ultrarelativistic charged particles through the water. The produced signals are then sent to the data acquisition electronics where they are amplified, formatted, digitized and stored. The data are recorded in single and triple coincidence modes.
\end{abstract}

35th International Cosmic Ray Conference - ICRC2017

10-20 July, 2017

Bexco, Busan, Korea

${ }^{*}$ Speaker. 


\section{Introduction}

The Laboratory for the Instrumentation of High Energy Radiation Detectors (LIDRAE ${ }^{1}$ ) [1] [2] is an array of 3 water-Cherenkov tanks designed to detect cosmic-ray shower particles at the ground level. LIDRAE is located in the campus of the Federal University of ABC (UFABC), in the city of Santo André (23.6 S, $46.5^{\circ} \mathrm{W}, 750 \mathrm{~m}$ a.s.l.). The tanks are buried in a garden, forming a triangular array whose sides are slightly larger than $8 \mathrm{~m}$ (see figure 1).

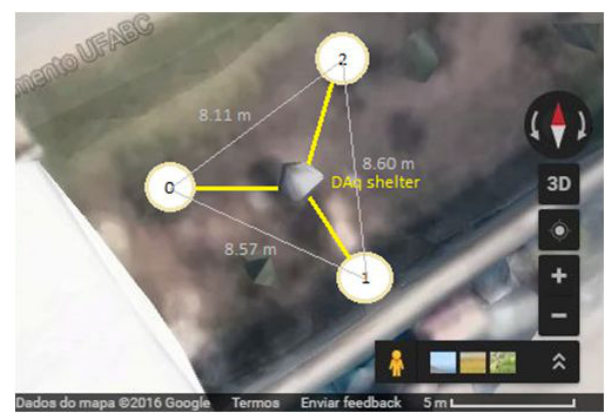

Figure 1: Layout of the LIDRAE array [3].

The detectors consist of tanks of $70 \mathrm{~cm}$ height and $134 \mathrm{~cm}$ diameter filled with one thousand liters of water. Each tank is equipped with a large aperture PhotoMultiplier Tube (PMT) of 8" diameter (Hamamatsu R5912 [4]) on its top cover (see figure 2). The high-voltage and signal cables pass underground, reaching a central shelter where the Data Acquisition (DAq) system is installed (see figure 1). A schematic view of the LIDRAE's DAq system is shown in figure 3. The signals from each detector (det) are sent to amplifiers (amp), where their amplitudes are amplified by a factor of 10, and to current-sensitive discriminator (dsc) modules, which select pulses with amplitudes higher than $\sim 60 \mathrm{mV}$. The formatted pulses are then sent to a logic box, which looks for triple coincidences (coinc) within a time windows of $50 \mathrm{~ns}$. Triple coincidence signals are used to start a time-to-digital converter (TDC), and are used as gates to a charge-to-digital converter (QDC), and are counted by a scaler. The discriminated signal from each detector is used to stop its TDC channel, and the analog signal from each detector is integrated in the QDC within the open gate. Finally, a third discriminator output (not shown in figure) from each channel is used to count the detectors trigger rates at every $10 \mathrm{~s}$. The data are collected through the VME bus. The trigger rate for each tank is of about $35 \mathrm{~Hz}$, and for triple coincidence is of about $0.025 \mathrm{~Hz}\left(\sim 1.5 \mathrm{~min}^{-1}\right)$.

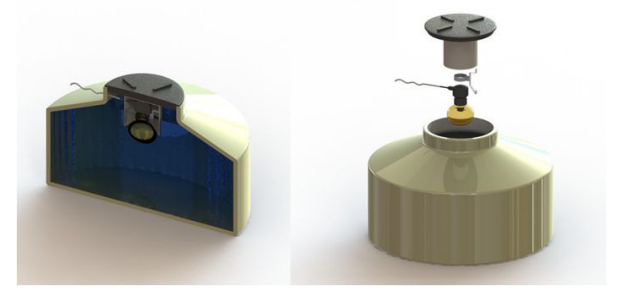

Figure 2: Lateral cut (left) and exploded view (right) of a LIDRAE tank.

\footnotetext{
${ }^{1}$ Acronym in Portuguese for "Laboratório para a Instrumentação de Detectores de Radiações de Altas Energias".
} 

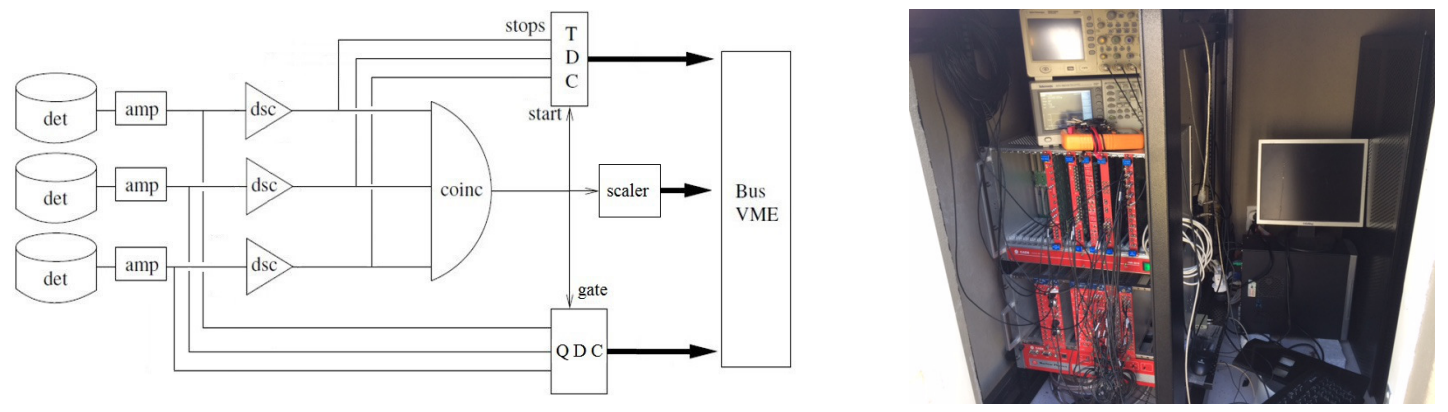

Figure 3: A block diagram for the DAq system of the LIDRAE array (left); and the actual setup (right).

\section{Setup calibration}

We first performed the calibration of QDC module (CAEN V965a [5]). Pulses generated at the frequency of $1 \mathrm{kHz}$ were sent to a dual gate generator (CAEN V462 [5]) of NIM pulses. A first output was used as the QDC gate, and another output to restart the dual gate generator, whose output had its width varied, consequently its charge, and was subsequently sent to a QDC channel to be converted with 12 bit resolution for two simultaneous ranges: $100 \mathrm{pC}$ (low) and $800 \mathrm{pC}$ (high). See figure 4 for the circuit of this calibration and the results for a given QDC channel. A VME bus controller (CAEN V1718 [5]) was used to run data acquisition.
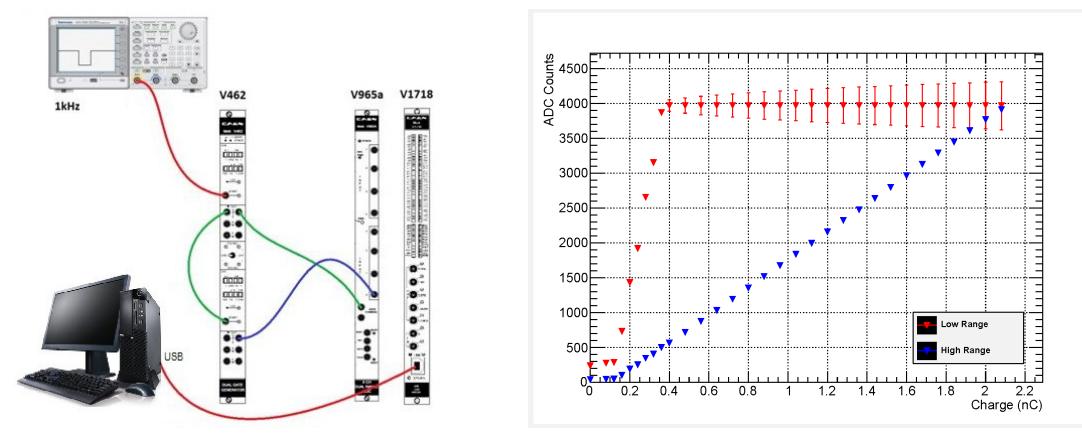

Figure 4: Setup for the calibration of the QDC module (left). Results for QDC channel 0 (right).

We then performed the characterization of the PMT signals in a dark chamber designed for that purpose: a PVC cylinder with $300 \mathrm{~mm}$ diameter was sealed against light entry to house the PMT to be tested, and a LED driven by a pulse generator of variable-pattern signals (see figure 5). We fed the PMTs with $1000 \mathrm{~V}$ given by a high-voltage power supply (CAEN N472 [5]). The anode signal was directed to the QDC module (CAEN V965A [5]) that received as gate a NIM-formatted pulse from a pulse generator, synchronized with another identical generator that supplied voltage to the LED. We collected data for different pulse widths applied to the LED, i.e. varying the flash time duration, and consequently the charge of the anode signal generated by the PMT. This setup has enabled us to determine the operating limits of each PMT in conjunction with the QDC module. The results for a given PMT are presented in right panel of figure 5, the other two PMTs had similar results. We can observe the intervals within which we must work, where the ADC counts vary with the pulse width for its two ranges of integration (low and high). 

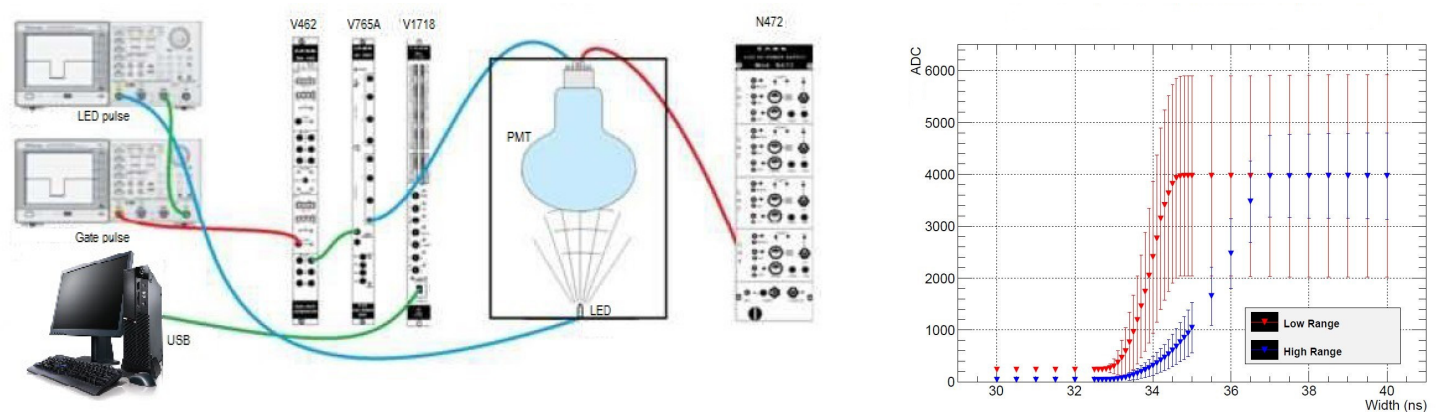

Figure 5: Setup for the calibration of the PMTs (left). Results for a given PMT (right).

We finally performed the calibration of the TDC module (CAEN V775N [5]) by sending two known formatted signals, one delayed from another by previously measured time intervals with the use of an oscilloscope. The first signal was used as the TDC starting pulse and the delayed one as the TDC stopping pulse. In figure 6, we present the results for three TDC channels. As we can see, there is a good linearity between $20 \mathrm{~ns}$ and $200 \mathrm{~ns}$. The resulting interval of linearity is compatible with separations between the tanks ranging from 6 up to $60 \mathrm{~m}$, considering the extreme case for horizontal showers triggering the array.
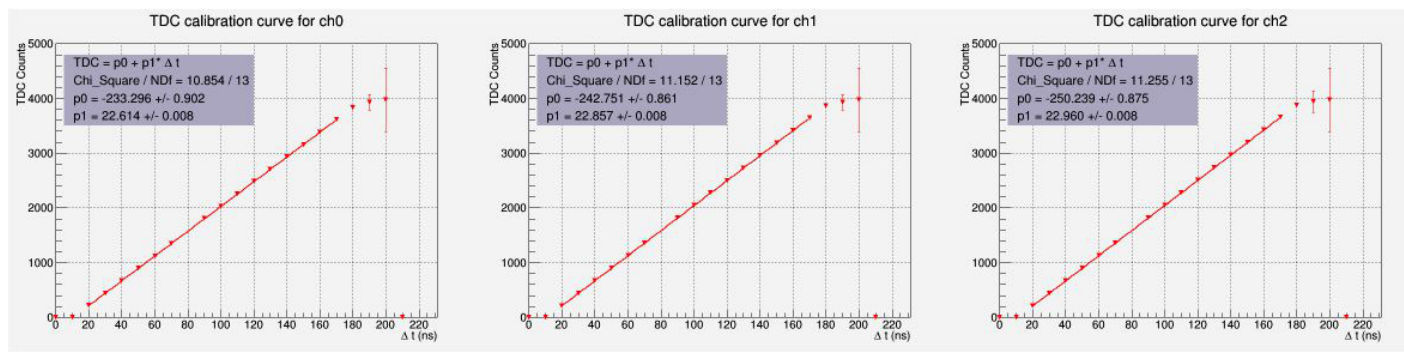

Figure 6: Calibration curves for the TDC channels 0,1 , and 2.

\section{Count rates}

After the steps of calibration and characterization of the electronics, and the PMTs, the array has been turned on and is continuously taking data. An important test is the monitoring of the signals count rates for the detectors under self-triggering mode. It is important for understanding the detectors stabilities, or their variabilities with atmospheric conditions, as well as with the flux of muons. In figure 7, we present the results for the countings after some days of data taking under self-triggering regime. One can easily see the variations on the average count rates which nicely correlate with the air-pressure daily cycle.

The signals from water-Cherenkov detectors originate mainly from muons — and a small part of electrons (and positrons) - of both charges crossing the water at relativistic speeds with varying zenith angle. At the altitudes of LIDRAE $\left(947 \mathrm{~g} / \mathrm{cm}^{2}\right)$, muons are the dominant contribuition to the flux of secondary cosmic rays with about $100 \mathrm{~m}^{-2} \mathrm{~s}^{-1} \mathrm{sr}^{-1}$ [6]. Since we report single-tank count rates of $35 \mathrm{~Hz}$, we estimate an efficiency of about $8 \%$ for secondary muons above $250 \mathrm{GeV}$ [7]. 


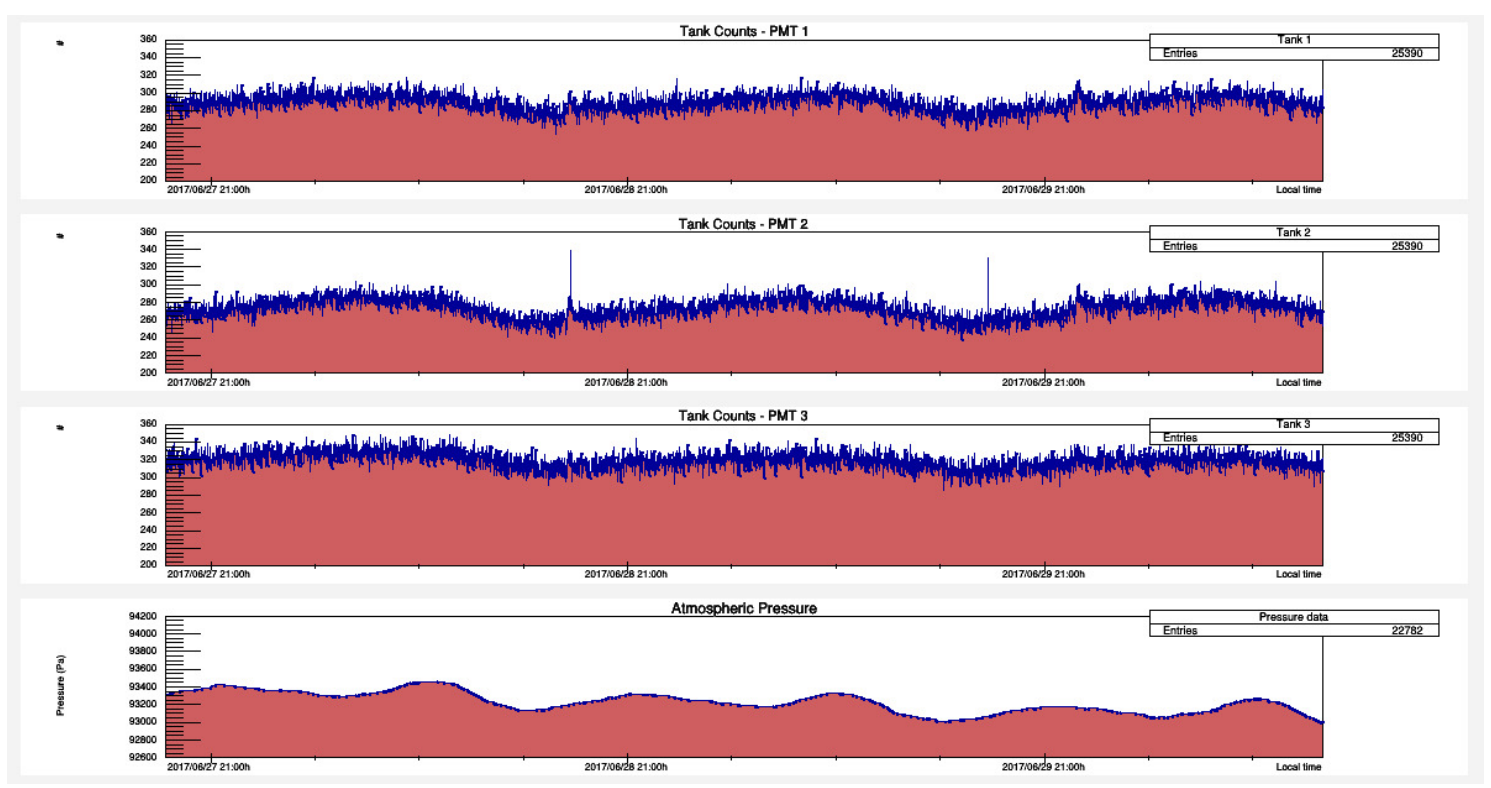

Figure 7: Count rates of the 3 LIDRAE tanks during 3 days of data taking under self-triggering regime.

\section{LIDRAE events}

LIDRAE has been taking data continuously since March, 2016. On April 18th, 2016, at 00:39 UT, the sunspot AR2529 erupted, producing a strong solar flare, classified as M6.7-class [8]. NASA's Solar Dynamics Observatory recorded the flare's extreme ultraviolet flash as a pulse of UV radiation that ionized the top of Earth's atmosphere (see figure 8).

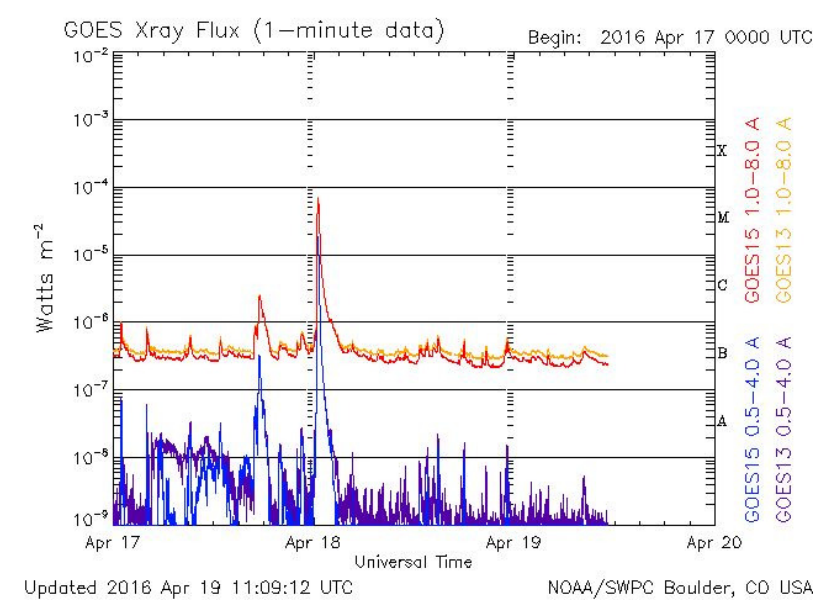

Figure 8: X-ray flux recorded by NASA's Solar Dynamic Observatory on April 18th, 2016 [9].

Some hours later, on the same day, a large increase in the LIDRAE array count rates was observed (see figure 9). They appeared as many consecutive peaks, whereas one of them lasted for $1 \min 25 \mathrm{~s}$. The highest one (central in figure 9) reached the level of $30 \sigma$ in the triple coincidence, if compared with the previous baseline count rate. 


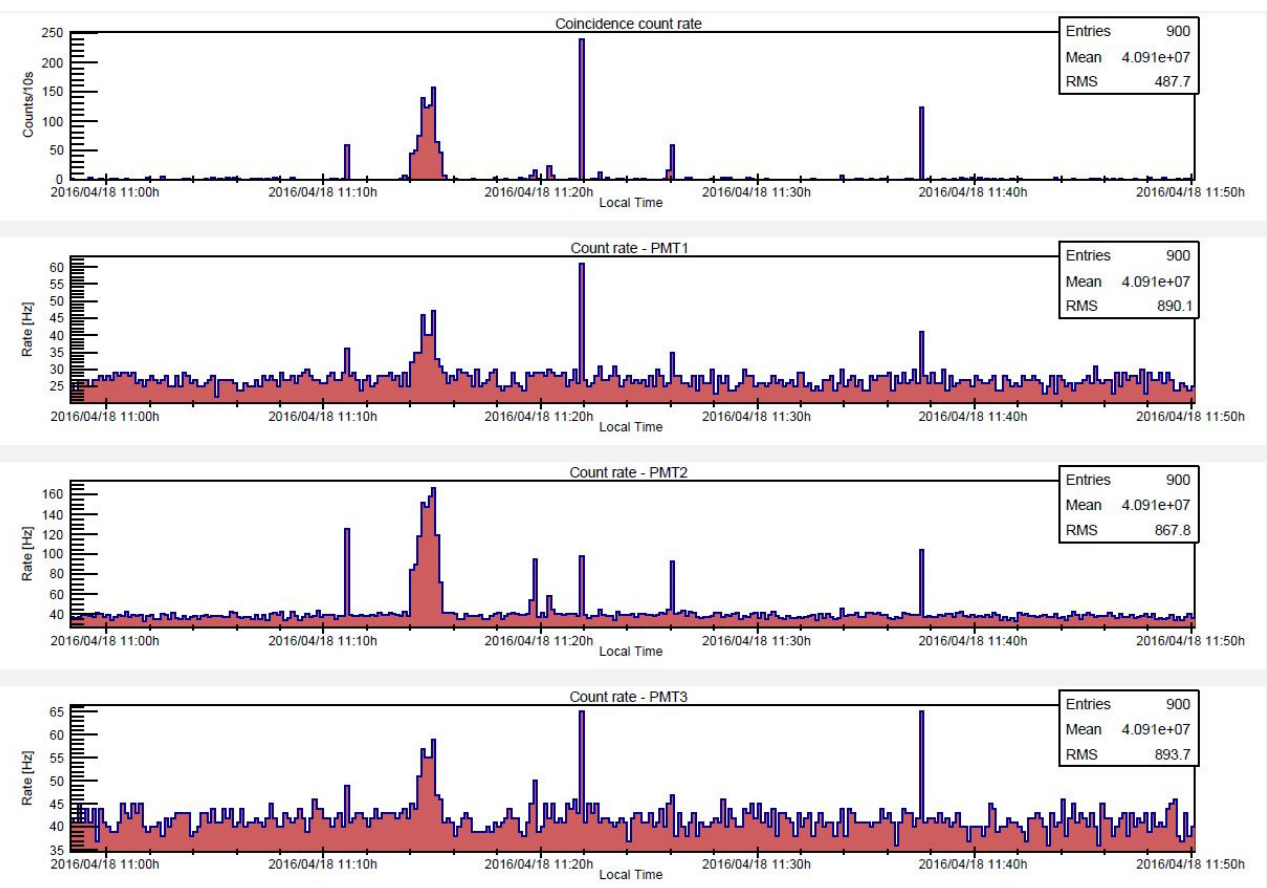

Figure 9: LIDRAE scaler count rates for the triple coincidence (upper panel) and for the single tanks (following panels) on April 18th, 2016, after 11:00 local time.

Since that detection, several other events have been observed with the same characteristics: on July 4th, and 22nd, August 9th, October 11th, November 30th, and December 16th, 2016, and January 23rd, March 9th, 21st, and 29th, 2017. On October, 2016, we also implemented pulses driven by a generator with the same frequencies of the single tanks going through the DAq. The intention was to verify if some electronic noise could be the cause of these observations, but no increase in the false-pulses count rates have been seen during the same instants of the recorded events (see figure 10).

All of these events present similarities: large increases of the count rates, large duration (from several seconds up to a few minutes), they have happened in hours when the LIDRAE array was on the daylit side of our planet, and the Sun was in periods of high activity. These events may be compatible with a train of low-energy particles of solar origin impinging on the top of the atmosphere. Further analyses are ongoing.

\section{Conclusion}

We presented the design, and the DAq system of the the LIDRAE water-Cherenkov airshowers array installed at UFABC. The array is composed of three tanks filled with one thousand liters of water equipped a large aperture photomultiplier on the top cover of each tank detecting the Cherenkov photons generated in water by the passage of secondary cosmic-ray charged particles. The signals are recorded in single and triple coincidence modes. A first analysis of the recorded events in scaler mode has been presented, and we reported events with large increases of the count rates, and large duration that could have relation with solar flares. 


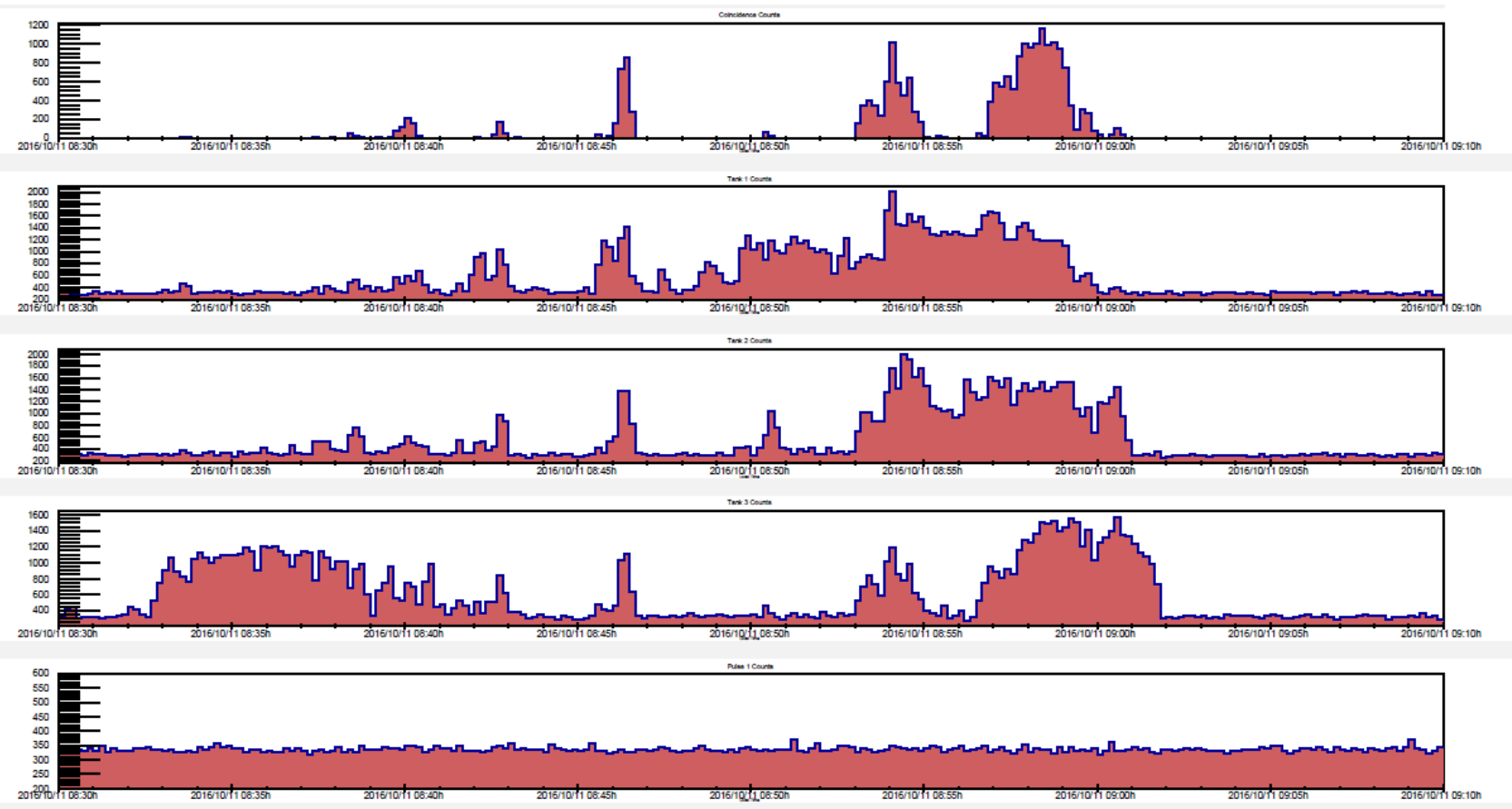

Figure 10: LIDRAE scaler count rates for the triple coincidence (upper panel), for the single tanks (following 3 panels), and for the false pulse (lower panel), on October 11th, 2016, after 8:30 local time.

\section{Acknowledgments}

This work has been supported by FAPESP (The São Paulo State Research Foundation), research grant 2008/00879-4, and by UFABC to whom the authors are very thankful.

\section{References}

[1] M. A. Leigui de Oliveira, Instrumentação de um Laboratório de Altas Energias e Análise de Dados do Observatório Pierre Auger, FAPESP (Grant Number 2008/00879-4).

[2] V. P. Luzio, M. A. Leigui de Oliveira, and R. Q. da Silva, Instrumentation of a Cherenkov tank for the project LIDRAE, Proc. of the 33rd ICRC, Rio de Janeiro (2013), CR-IN, ID= 1219.

[3] Figure adapted from Google Maps, https://maps.google.com.br

[4] Hamamatsu Photonics, http://www.hamamatsu.com

[5] CAEN - Electronic instrumentation, Products Catalog (2007), http://www.caen.it

[6] C. Patrignani et al. [Particle Data Group], Review of Particle Physics, Chin. Phys. C, 40, 100001 (2016) 423.

[7] P. K. F. Grieder, Cosmic Rays at Earth, Elsevier, Amsterdam (2001), $1^{\text {st }}$ ed., pp. 236.

[8] http://spaceweather.com/archive.php?view=1\&day=18\&month=04\&year=2016

[9] http://www.polarlicht-vorhersage.de/goes_archive 